\title{
Lanadelumab Injection Treatment For The Prevention Of Hereditary Angioedema (HAE): Design, Development And Place In Therapy
}

This article was published in the following Dove Press journal:

Drug Design, Development and Therapy

\author{
Maria Bova $\mathbb{D}^{\prime}$ \\ Anna Valerieva ${ }^{2}$ \\ Maddalena Alessandra Wu (iD) ${ }^{3}$ \\ Riccardo Senter (iD) 4 \\ Francesca Perego (iD ${ }^{5}$ \\ 'Department of Translational Medical \\ Sciences and Interdepartmental Center \\ for Research in Basic and Clinical \\ Immunology Sciences, University of \\ Naples Federico II, Naples, Italy; ${ }^{2}$ Clinical \\ Centre of Allergology, Medical University \\ of Sofia, Sofia, Bulgaria; ${ }^{3}$ Department of \\ Biomedical Science, University of Milan, \\ Milan, Italy; ${ }^{4}$ Department of Medicine, \\ University of Padova, Padova, Italy; \\ ${ }^{5}$ Department of Cure Subacute, Istituti \\ Clinici Scientifici Maugeri, IRCCS, Milan, \\ Italy
}

\begin{abstract}
Despite the efficacy of the on-demand treatment for the control of acute attacks of Hereditary Angioedema due to C1-Inhibitor Deficiency (C1-INH-HAE), the number and severity of attacks and the impairment in the quality of life of the affected patients have led to the development of a new monoclonal antibody, lanadelumab, directly addressed to the blockage of bradykinin, the principal mediator of vasodilation during angioedema attacks. It is indicated for the prophylactic treatment, it is easy to administer, highly effective and with known limited side effects. The current review summarizes the development of the drug, its clinical background and its perspectives.
\end{abstract}

Keywords: C1-inhibitor hereditary angioedema, long-term prophylaxis, lanadelumab, monoclonal antibody, rare disease

\section{Plain Language Summary}

For the patients affected by hereditary angioedema, the features of the ideal drug for the treatment of angioedema attacks are to be easy to assume in the terms of time interval between two treatments, have an easy route of administration, reach a complete effectiveness in the control of attacks and be burdened by limited side effects. In the last years the interest towards the development of new drugs was rapidly increasing. Lanadelumab is a fully human, $\kappa$-lightchain, Chinese hamster ovary cells expressed monoclonal immunoglobulin, a highly potent and selective antibody against Plasma Kallikrein (PK) developed to be used for long-term prophylaxis of attacks in patients with a severe phenotype of the disease. It has been tested in a double-blind placebo-controlled single-center Phase Ia study by a single subcutaneous injection. Lanadelumab prevented proteolysis of High Molecular Weight Kininogen and determined PK inhibition with biological effects detectable until 20 days after dosing. The Phase Ib study had the primary objectives to assess safety, pharmacokinetic and pharmacodynamic parameters and antidrug antibodies in HAE patients and, secondarily, to assess its efficacy in reducing the frequency of attacks. In the Phase III Hereditary Angioedema Long-term Prophylaxis (HELP) clinical trial all lanadelumab different treatment regimens tested were more effective than placebo. No notable safety concerns have been registered throughout the clinical development program.

\section{Introduction}

Hereditary angioedema with $\mathrm{C} 1$ esterase inhibitor (C1-INH-HAE) deficiency is characterized by recurrent, not erythematous, circumscribed, sudden and unexpected swelling of the cutaneous and subcutaneous tissues involving face, larynx
Correspondence: Francesca Perego Department of Cure Subacute, Istituti Clinici Scientifici Maugeri, IRCCS, via Camaldoli 64, Milan 20138, Italy Tel +393489050440

Email francesca.perego@icsmaugeri.it 
and gastrointestinal tract. ${ }^{1}$ It can be disabling and disfiguring, and could be potentially life-threatening, above all when upper airways are involved. C1-INH-HAE is a rare autosomal dominant disease, resulting in attacks of acute edema, that occurs in members of the same family. The development of swelling is slow (hours) and complete spontaneous remission occurs in 72-96 hrs.

In spite of the growing attention that medical community and all the stakeholders have paid in the last decades to HAE patients, the burden of this disease is very heavy. $^{2,3}$

The disease impact on daily-life not only during attacks, but also in the free from attack time, worsens the psycho-social life, expectations and productivity of these patients in the school/work world. ${ }^{3}$

The expression of the disease is very different between patients, varying from patients with high frequency and severe disease to very mild phenotypes. ${ }^{4}$ Severe patients need to be treated not only during acute attacks, but also demand for an efficacious and safe prophylactic treatment. ${ }^{1}$ The decision on when to associate a prophylactic treatment to on-demand therapy is still difficult and is based on different aspects related to the frequency of attacks, localization, quality of life of the patients, availability of treatment, route of administration, safety profile and evaluation of cost-efficacy. Current prophylaxis treatment is all perfectible. In spite of the widespread availability of HAE medications in many countries, HAE patients' quality of life is still quite low and there are numerous unmet needs. ${ }^{5}$ Moreover, due to the high costs of specific HAE drugs, depending on the healthcare system, in some countries, there are invincible barriers to obtain treatment for many patients. ${ }^{6-8}$

In this context, many scientists are working on new and better prophylactic drugs. Lanadelumab is a fully human monoclonal antibody that specifically inhibits PK.
Lanadelumab has demonstrated a good efficacy in HAE prophylaxis and safety profile, and seems to be able to dramatically improve the quality of life of patients with severe phenotypes. Its specific characteristics, like the subcutaneous administration and the infrequent dosing, may help to solve some of the limitations of existing prophylactic options. Introduction of lanadelumab on a large scale could dramatically change the current treatment paradigm.

In this paper we will elaborate on the details of the design and development of lanadelumab, focusing on its place in the current and future HAE therapy.

\section{Genotypes And Phenotypes Of CI-INH-HAE}

C1-INH-HAE is a rare disease with a quite uniform prevalence worldwide. A recently published systematic investigation summarizes the numerous country-based data on this topic, estimating that $\mathrm{C} 1-\mathrm{INH}-\mathrm{HAE}$ prevalence varies between 1.1 and 1.6 per $100.000 .^{9}$

In most patients with HAE, there are mutations in the $\mathrm{C} 1$ inhibitor gene (SERPING1), which result in the decreased synthesis of normal C1-INH (C1-INH-HAE type I) or expression of not functional C1-INH (C1-INH-HAE type II). However, mutations in the gene coding for coagulation Factor XII have been identified in a subset of patients who present familiar recurrent episodes of $\mathrm{AE}$, but no abnormalities in complement parameters and no mutation in the SERPING1 gene (FXII-HAE). ${ }^{10}$ Recently novel mutations in angiopoietin-1 (ANGPT1), plasminogen (PLG) and kininogen 1 (KNG1) genes have been associated with $\mathrm{HAE}^{11-13}$ There are also many families with clear hereditary AE and no genetic mutation identified so far and this variant is currently defined as unknown HAE (U-HAE) ${ }^{1}$ (Figure 1).

The Hereditary Angioedema Working Group (HAWK) classification of angioedema without wheals ${ }^{1}$ also describes acquired forms of $\mathrm{AE}$, some of them potentially

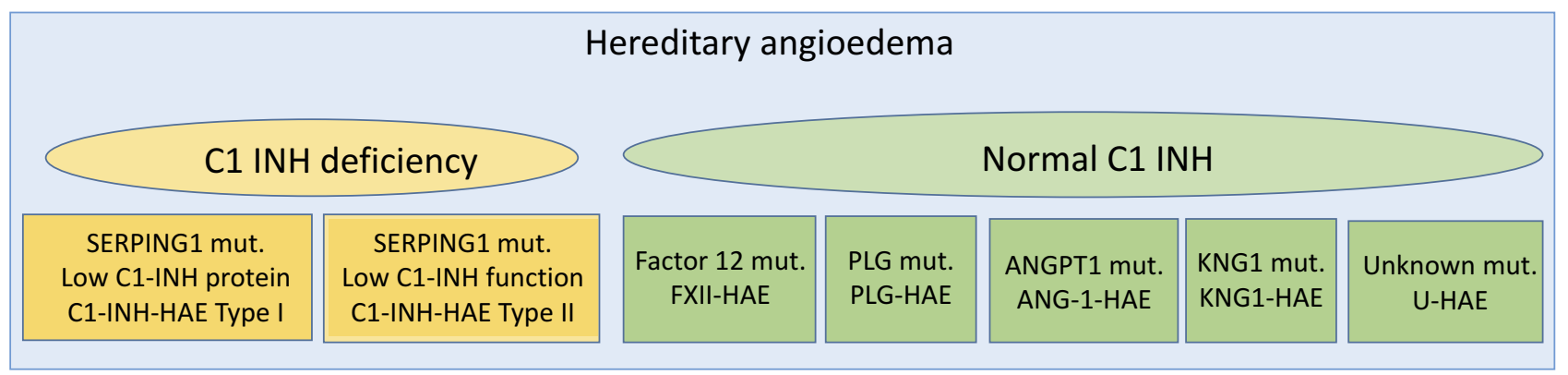

Figure I Classification of hereditary angioedema.

Abbreviations: $\mathrm{CI}-\mathrm{INH}, \mathrm{Cl}$ inhibitor; HAE, hereditary angioedema; FXII, factor XII; PLG, plasminogen; ANGI, angiopoietin I; KNGI, kininogen I; U, unknown. 
sharing pathogenetic characteristics with C1-INH-HAE angioedema. They are idiopathic non-histaminergic angioedema, acquired angioedema related to ace-inhibitor and the acquired form with $\mathrm{C} 1$ inhibitor deficiency.

The clinical spectrum of attacks, such as age of onset, localization, frequency and severity, differs among patients affected by HAE. Patients may also present a significant variability of the frequency of attacks during time, not always referable to identifiable factors and without any correlation between genotype and phenotype. ${ }^{14}$ Furthermore, frequency or severity of attacks may be worsened by stress, estrogen therapy or hormonal fluctuations, treatment with ACEI and by acute or chronic infections. Patients with so deeply different phenotypes require tailored therapeutic approach and have very specific needs.

\section{CI-INH-HAE Pathophysiology}

The complement system (CS) and contact activation systems (CAS) are complex and many molecules interact in an intricate play of roles, stimulating or blocking each other directly or indirectly. In the past decade, the look of researcher has been focusing on different patterns to identify potential target for new therapies.

Until just few decades ago, the only approach seemed to be the replacing of the lacking protein $\mathrm{C} 1-\mathrm{INH}$. It is the main inhibitor of the components of CS and a poor control of the local activation process of this system lead to bradykinin (BK) formation. The CAS consists of the proteins Factor XI, Factor XII, prekallikrein and high molecular-weight kininogen (HMWK). The first step in the activation of CAS is the production of the activated form of factor XII called FXIIa, which subsequently cleaves the prekallikrein to generate kallikrein. Kallikrein has two roles: it activates additional FXII and produces BK and cleaved HMWK from HMWK. BK is essential in the pathogenesis of $\mathrm{C} 1-\mathrm{INH}-$ $\mathrm{HAE}$ and represents the end product of CAS and kallikreinkinin system. BK is a vasoactive nonapeptide, acting on two different types of receptors present on endothelial cells: B1R, an inducible receptor, and $\mathrm{B} 2 \mathrm{R}$, expressed constitutively on the cell surface. BK binds the B2R and activates various proinflammatory signaling pathways increasing vascular permeability. High levels of $\mathrm{BK}$ are detectable in plasma of patients with HAE due to $\mathrm{C} 1-\mathrm{IHN}$ deficiency during the attacks. ${ }^{14}$ In the last decades research, efforts were concentrated about the pattern BK-BR receptor, bringing to a completely different approach for the therapy of acute attacks with icatibant, ${ }^{15}$ a molecule blocking BK receptor.
Nowadays kallikrein, for his well-recognized key role in the pathogenesis of C1-INH-HAE, was identified like a targeting molecule in the pharmacologic management of this disease.

The role of kallikrein in the primary angioedema with normal C1-INH (Figure 1) is more controversial. In HAE due to FXII mutation ${ }^{10,16}$ there is an enhanced susceptibility of the CS to become activated both ex vivo and in vivo. ${ }^{17}$ The principal mediator of this type of angioedema remains BK via an enhanced activation of mutant FXII by plasmin and enhanced production of plasma kallikrein by FXIIa. In ANGPT1-HAE ${ }^{18}$ there is an impaired ability to control vascular permeability, with a mechanism still unclear but that does not possibly involve the Kallikrein-BK pathway. In PLG-HAE, ${ }^{19}$ the final angioedema mediator could remain BK after an enhanced activation of mutant plasminogen by tissue plasminogen (tPa) and/or urokinase plasminogen activator (uPA). Another form of HAE with normal C1-INH has recently been discovered by Bork et al. ${ }^{13}$ In this case, the enhanced activation of mutant HMWK by kallikrein leads to an increased production of BK or, as an alternative hypothesis, the production of functional active but aberrant BK, with decreased susceptibility to inactivation by enzymes could lead to a reduced catabolism of BK (Figure 2). In addition, there are forms of HAE not yet characterized without identified mutations called U-HAE.

\section{Current Therapeutic Landscape}

Management of C1-INH-HAE is based on the control of acute attacks and, when necessary, on the long-term prophylactic treatment (LTP), whose goal is lowering frequency and severity of attacks. Before invasive procedures or potentially attacks triggering situations, patients are also advised to use short-term prophylaxis (STP). ${ }^{1}$

Based on local availability, and with deep differences between countries, there are three therapeutic options for acute attacks, which target different steps of the pathway from kallikrein to $\mathrm{BK}$ production and action. Plasmaderived C1-INH (pdC1-INH-Berinert ${ }^{\circledR}$, CSL Behring or pdC1-INH-Cinryze ${ }^{\circledR}$, Shire) and recombinant C1-INH (rhC1-INH-Ruconest, ${ }^{\circledR}$, Pharming Group NV) are aimed to replacing the $\mathrm{C} 1$ inhibitor; $^{20}$ Icatibant (Firazyr $^{\circledR}$, Shire) blocks the bradykinin B2 receptor, ${ }^{15}$ and ecallantide (Kalbitor $^{\circledR}$, Dyax), not yet available in Europe, that reversibly inhibits the enzyme kallikrein. ${ }^{21}$

LTP aims to reduce frequency, duration and severity of episodes in patients affected from C1-INH-HAE, especially in those with severe phenotypes, or when recommended 


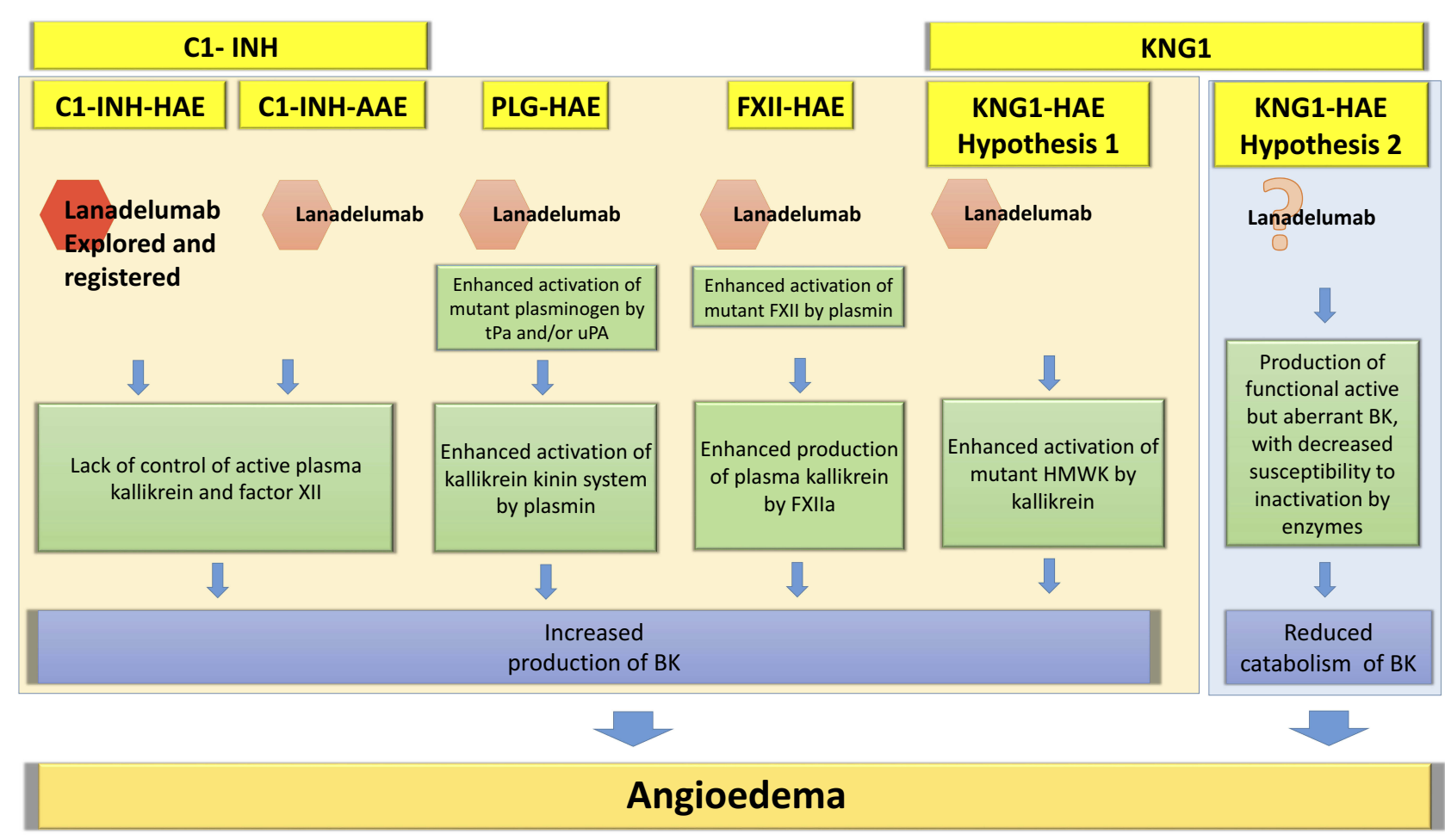

Figure 2 Hypothesis for a potential role of lanadelumab in hereditary angioedema with normal $\mathrm{Cl}$ inhibitor and acquired angioedema with $\mathrm{Cl}$ inhibitor deficiency. Notes: Pathogenesis of hereditary angioedema with normal $\mathrm{Cl}$ inhibitor is partially known. This figure is based on speculation about the possible mechanisms hypothesized for these diseases. In KNGI-HAE, we report the two pathogenetic hypothesis described by Bork et al Allergy 20I9. In hypothesis I, we imagine a possible role of lanadelumab, while in the hypothesis 2 , based on a reduced catabolism of BK, we put a question mark. We did not include ANGPTI-HAE because the few data considered about its pathogenesis suggest a different mechanism not including the kallikrein-kinin system. In spite of this, we keep open new possibilities, based on the described efficacy of tranexamic acid in this group of patients. *Lanadelumab is only explored and registered for prophylactic treatment of $\mathrm{CI}-\mathrm{INH}-\mathrm{HAE}$ patients.

Abbreviations: BK, bradykinin; HMWK, high molecular weight kininogen; tPa, tissue PLG activator; uPA, urokinase PLG activator; PLG-HAE, hereditary angioedema with mutation in plasminogen (PLG) gene; KNGI-HAE, hereditary angioedema with mutation in kininogen I gene; FXII-HAE, hereditary angioedema with mutation in FXII gene (FI2); $\mathrm{CI}-\mathrm{INH}-\mathrm{AAE}$, angioedema with acquired $\mathrm{Cl}$-inhibitor deficiency.

drugs are difficultly available. Danazol and other attenuated androgens (AA) (stanozolol, oxandrolone) used to be the most common prophylactic drugs in past years. ${ }^{22}$ Their potential multiple dose-related adverse events have limited the use of these drugs in some countries. Some of the adverse events like virilization, weight gain and menstrual irregularities make AA particularly unpopular in the women group. PdC1-INH concentrate could be administered prophylactically twice weekly e.v. (Cinryze ${ }^{\circledR}$ ViroPharmaInc, Exton, PA, USA) or s.c. (Haegarda). RhC1-INH is also been proved efficacious for HAE prophylaxis. ${ }^{23}$

Several studies are now focusing on blocking kallikrein to treat angioedema. BCX7353 is an orally administered kallikrein inhibitor with a potential role in the treatment of oral attacks and prophylaxis of HAE. ${ }^{24}$ KVD 900 [https://www.clinical-trials.gov] is a selective inhibitor of Pka, an oral treatment with a possible task in the treatment of acute attacks, and finally ATN-249, a novel oral plasma kallikrein inhibitor, that could be useful for prophylactic use in prevention of HAE attacks. Other ongoing animal experimental model are approaching HAE treatment based on new targets, like factor XIIa, prekallikrein and genetic therapy. ${ }^{25-28}$

The pipeline of C1-INH-HAE therapy is very promising for the improvement of C1-INH-HAE patients' health condition.

In the next paragraphs the design, development and place in therapy of lanadelumab will be discussed.

\section{Molecular Features And Preclinical Studies}

DX-2930, later called lanadelumab, is a fully human, $\kappa$-light-chain, Chinese hamster ovary cells expressed monoclonal immunoglobulin $\mathrm{IgG1}{ }^{29}$ It was created using the phage display technology ${ }^{30}$ with the purpose to build a 
highly potent and selective antibody against Plasma Kallikrein (PK). In fact, the drug shows an optimal inhibition constant $\left(\mathrm{K}_{\mathrm{i}}=120 \pm 5 \mathrm{pM}\right)^{29}$ and does not interfere with several other serin-proteases, such as the closely related Factor XIa and the zymogen prekallikrein. ${ }^{29}$ The absence of inhibiting action against the zymogen prekallikrein was deliberately intended, in order to reduce the amount of drug needed to inhibit $\mathrm{PK} .{ }^{29}$

Lanadelumab binds to the PK active site, occluding it completely, as shown in $2.1-\AA$ resolution crystal structure analysis; ${ }^{29}$ moreover, Western blot assessment shows that the drug is not proteolyzed. Lanadelumab blocks the active site when PK is free in the plasma, when it is bound to HMWK and when PK-HMWK complexes are adherent to the surface of human umbilical endothelial cells. ${ }^{29}$

In vivo pharmacokinetic and pharmacodynamic analyses were initially conducted in Cynomolgus Monkeys, where a half-life of 12.5 days post single subcutaneous 20 $\mathrm{mg} / \mathrm{kg}$ dose was found. The proteolysis of HMWK was prevented for 28 days after dosing as demonstrated by Western blot assay. ${ }^{29}$

The first evidence of in vivo efficacy derived from a rat model of unspecific, multiple mediators-driven inflammation, the carrageenan-induced paw edema: dose-dependent reduction of edema was observed in lanadelumab premedicated rats in comparison with vehicle-treated animals. ${ }^{29}$

The results of the preclinical studies supported the following development of the drug in the clinical setting.

\section{Phase la Study}

The Phase Ia study, a double-blind placebo-controlled single-center trial, included 32 healthy subjects (age 18-55 years) randomized to receive a single subcutaneous injection of either lanadelumab or placebo within ascending dose cohorts $(0.1,0.3,1$, or $3 \mathrm{mg} / \mathrm{kg}) .{ }^{31}$ The ratio between lanadelumab and placebo was $3: 1$; subjects were followed for 16 weeks after dosing. Two subjects did not complete the study for reasons not related to adverse effects.

Lanadelumab prevented proteolysis of HMWK when administered at the dose of $3 \mathrm{mg} / \mathrm{kg}$, as demonstrated by Western blot assessing the presence of cleaved HMWK; moreover, the drug determined PK inhibition at the dose of 1 and $3 \mathrm{mg} / \mathrm{kg}$ as confirmed by Kallikrein functional analysis. These biological effects remained detectable 20 days after dosing. ${ }^{31}$

Mean maximum plasma concentrations were proportional to the dose $(14.5 \mu \mathrm{g} / \mathrm{mL}$ for the $3 \mathrm{mg} / \mathrm{kg}$ cohort), while mean elimination half-life was similar among the different doses (range 16.8-21.2 days). ${ }^{31}$

\section{Phase Ib Study}

The Phase Ib study, a randomized, double-blind, placebocontrolled, multicenter trial had the primary objectives to assess safety, pharmacokinetic and pharmacodynamic parameters and antidrug antibodies in HAE patients treated with Lanadelumab, and, secondarily, to assess its efficacy in reducing the frequency of attacks. ${ }^{32}$

Relevant eligibility criteria were: at least 2 attacks in the previous year, one of which in the past 6 months; no LTP in the past 90 days; no replacement treatment with $\mathrm{C} 1-\mathrm{INH}$ in the previous week; no treatment with any monoclonal antibodies in the past 5 years. On-demand treatment was allowed.

Thirty-seven patients, aged 18-71 years (mean age 39.9 years), were randomized to a specific lanadelumab dose $(30,100,300$ or $400 \mathrm{mg})$ or to placebo; the ratio between lanadelumab and placebo was 2:1. Each patient underwent two subcutaneous injections, separated by 14 days.

Efficacy analysis was conducted on the patients of the higher lanadelumab dose groups (300 and $400 \mathrm{mg}$ ) who had at least 2 attacks in the previous 3 months. Only attacks occurred between day 8 and day 50 were considered. Nineteen patients were included; an overall 91\% reduction of attacks was found, compared with the patients in the placebo group. Only one attack occurred in the period of predicted drug efficacy. ${ }^{32}$ One patient was lost to follow-up after the first attack; another one was excluded a posteriori because the diagnosis of $\mathrm{Cl}$-inhibitor deficiency angioedema was questioned. Post-hoc analysis, which excluded these two patients, yielded an even stronger result ( $97 \%$ of reduction of the attacks).

Lanadelumab prevented the proteolysis of HMWK at the dose of 300 and $400 \mathrm{mg}$, as demonstrated by Western blot assessing the proportion of cleaved HMWK. Moreover, lanadelumab at the dose of 100, 300 and 400 mg significantly inhibited PK as confirmed by kallikrein functional analysis. Lanadelumab effect was dose-dependent in all the cases. ${ }^{32}$

The pharmacokinetic analysis showed a similar mean half-life across all the groups (range 13.8-15 days), with mean maximum plasma concentrations proportional to the dose $(45.3 \mu \mathrm{g} / \mathrm{mL}$ for the $400 \mathrm{mg}$ cohort $){ }^{32}$

Overall, these findings supported the assessment of lanadelumab in larger trials. 


\section{Phase III Trial}

The Hereditary Angioedema Long-term Prophylaxis (HELP) clinical trial ${ }^{33}$ was designed to investigate the efficacy, adverse events, and other safety parameters of subcutaneously administered lanadelumab compared with placebo for preventing HAE attacks. It was a Phase 3, randomized, double-blind, parallel-group, placebo-controlled trial conducted at 41 sites in Canada, Europe, Jordan, and the United States.

Patients 12 years or older with HAE type I or II underwent a 4-week run-in period (preceded by a $\geq 2$-week washout of any long-term prophylactic therapy, if applicable) to determine their baseline attack rate. Patients with 1 or more investigator-confirmed attack per 4 weeks were enrolled and randomized 2:1 to receive subcutaneously injected lanadelumab $(n=84)$ or placebo $(n=41)$, using an interactive web-based randomization system. Patients assigned to lanadelumab were further randomized 1:1:1 to 1 of the 3 dose regimens: $150 \mathrm{mg}$ every 4 weeks $(n=28)$, $300 \mathrm{mg}$ every 4 weeks $(\mathrm{n}=29)$, or $300 \mathrm{mg}$ every 2 weeks $(n=27)$. To maintain the blind, all patients received injections every 2 weeks, with those in the every-4-week group receiving placebo in between active treatments and every time 2 injections of the study drug were administered in the same upper arm. Over the 26-week treatment period (days 0-182), each patient received 13 doses of blinded study drug. At the end of the trial, patients could enter either an open-label extension study (HELP Study Extension, NCT02741596) or an 8-week safety followup. $^{34}$

Primary efficacy end point was the number of investigator-confirmed attacks of HAE over the treatment period.

During the trial, acute angioedema attacks were treated following the site investigator's standard of care (intravenous $\mathrm{C} 1 \mathrm{INH}$, icatibant or ecallantide).

Among 125 patients randomized (mean age, 40.7 years [SD, 14.7 years]; 88 females [70.4\%]; 113 white [90.4\%]), $113(90.4 \%)$ completed the study. During the run-in period, the mean number of HAE attacks ranged from 3.2 to 4.0 per month across the 4 treatment groups. During the treatment period, all lanadelumab treatment regimens were more effective than placebo. The mean number of attacks per month was 1.97 (95\% CI, 1.64-2.36) in the placebo group; for the lanadelumab groups, 0.48 (95\% CI, 0.310.73 ) in the $150-\mathrm{mg}$ every-4-week group, 0.53 (95\% CI, $0.36-0.77$ ) in the 300 -mg every-4-week group, and 0.26 (95\% CI, 0.14-0.46) in the 300-mg every-2-week group.
Therefore, the mean difference in attack rate per month in the lanadelumab groups vs the placebo group was -1.49 (95\% CI, -1.90 to -1.08$)$ in the $150-\mathrm{mg}$ every-4-week group, -1.44 (95\% CI, -1.84 to -1.04$)$ in the $300-\mathrm{mg}$ every-4-week group, and $-1.71(95 \% \mathrm{CI},-2.09$ to -1.33$)$ in the 300-mg every-2-week group (adjusted $\mathrm{p}<0.001$ for all comparisons).

Secondary end points included the number of attacks requiring acute treatment during the 26-week treatment period, number of moderate or severe attacks during the 26-week treatment period, and number of attacks from days 14 through 182. A statistically significant reduction in attack rates for all rank-ordered secondary efficacy analyses was detected for all lanadelumab groups as compared with placebo $(\mathrm{p}<0.001)$.

All lanadelumab treatment regimens were more effective than placebo also for additional prespecified exploratory end points, including the percentage of patients who were attack-free, number of attack-free days, responders, and number of high morbidity attacks. Moreover, they reported a significant improvement in quality of life total scores (as assessed through the Angioedema Quality of Life Questionnaire).

Of note, this study was powered to compare the efficacy of lanadelumab vs placebo but was neither designed nor powered to compare the effects of the 3 lanadelumab dose groups.

Exposure-response analyses showed that longer chronic treatment with lanadelumab $300 \mathrm{mg}$ every 2 weeks was associated with more marked improvement in HAE control (assessed as number of attacks per month) and significant delay in time to first attack. ${ }^{35}$

Subgroup analyses of data from the Phase 3 HELP Study highlighted that a significant reduction of HAE attack rate was observed in the treated group as compared with placebo regardless of age, gender, body weight and baseline HAE clinical characteristics. ${ }^{36}$

In order to evaluate the relationship between body weight, pharmacokinetics/pharmacodynamics and clinical endpoints, data from 84 patients treated with lanadelumab $150 \mathrm{mg}$ every 4 weeks, $300 \mathrm{mg}$ every 4 weeks or $300 \mathrm{mg}$ every 2 weeks for 6 months in the HELP Study were analyzed. The results of the analysis showed that lanadelumab plasma concentrations remained markedly above the IC50 across a large range of body weight (with associated cHMWK inhibition), particularly for the $300 \mathrm{mg}$ every 2 weeks regimen, with a good safety and tolerability profile. $^{37}$ Interim results from the HELP Open-Label 
Extension Study show that treatment with lanadelumab $300 \mathrm{mg}$ every 2 weeks led to a significant decrease of HAE attack rate compared with 3-month historical experience in patients with and without prior C1-INH long-term prophylaxis. ${ }^{38}$ Interestingly, patients who were "nonresponder" to lanadelumab $150 \mathrm{mg}$ every 4 weeks in the HELP Study experienced improvements with the higher dose of $300 \mathrm{mg}$ every 2 weeks in the HELP OLE Study. ${ }^{39}$

The efficacy and safety of lanadelumab for prophylaxis in C1-INH-HAE adolescents were demonstrated by a reduced monthly attack rate in patients between 12 and 18 years of age both in the Phase 3 HELP and in the HELP OLE Studies. ${ }^{40}$

\section{Safety And Tolerability Of Lanadelumab}

No notable safety concerns have been registered throughout the clinical development program of the drug since December 2013: a Phase I study ${ }^{31}$ in healthy individuals, one Phase Ib study ${ }^{32}$ in C1-INH-HAE patients, one completed Phase III trial (HELP study), ${ }^{33}$ and one ongoing Phase III open-label extension (HELP-OLE). ${ }^{34}$

\section{Phase la (Initiated 2013)}

In this single-center study, healthy subjects were randomized versus placebo to receive a single dose of lanadelumab $(0.1,0.3,1.0$, or $3.0 \mathrm{mg} / \mathrm{kg} / \mathrm{SC})$ in ascending dose cohorts. ${ }^{31}$ A total of 32 subjects were enrolled and randomized, of whom 30 completed the study (neither of the discontinuations were related to adverse events). No serious adverse events or death were reported. No clinically significant change in vital signs, physical status, or electrocardiogram was reported. No antidrug antibodies were detected. The most commonly reported adverse events in lanadelumab-treated patients were headache ( $25 \%$ vs $25 \%$ with placebo, none was severe, all resolved), and upper respiratory tract infections (3 subjects in doses $0.1,0.3$, and $1.0 \mathrm{mg} / \mathrm{kg} ; 12 \%$ vs $0 \%$ with placebo). No injection site adverse reactions were reported. ${ }^{31}$

\section{Phase la (Initiated 2018)}

As the majority of subjects exposed to treatment with lanadelumab throughout the clinical program were white, Caucasian subjects, a recent Phase I study (2018) in healthy volunteers evaluated pharmacokinetics (PK), safety, tolerability and pharmacodynamics (PD) of lanadelumab in healthy adult Japanese subjects and matched non-Hispanic healthy adult and Caucasian subjects. The study is completed and results wait to be reported (not the case by the date of manuscript preparation). [clinical trial.gov NCT03401671]

\section{Phase lb (Initiated 20I4)}

In this multicenter study, 37 C1-INH-HAE patients were randomized to receive 4 different doses of lanadelumab (30, 100, 300 , and $400 \mathrm{mg} / \mathrm{SC}$ ), or placebo (24 lanadelumab, 13 placebo). Within each dose group, patients received two doses of study treatment, 14 days apart. All patients completed the study, and no deaths were reported. No notable differences in adverse events were recognized between groups. A total adverse event incidence was $58 \%$ in lanadelumab treated vs $77 \%$ in the placebo group. Most common adverse reactions were attacks of angioedema, injection site pain (25\% lanadelumab vs $23 \%$ placebo), and headache ( $8 \%$ lanadelumab vs $15 \%$ placebo). Two patients who received lanadelumab had severe adverse events: one patient (30-mg group) had injection site pain for $1 \mathrm{~min}$, and the other had worsening of headache and night sweats. Another serious adverse event was pneumonia in the placebo group. Antidrug antibodies were detected in two patients. Antibodies were evaluated to be non-neutralizing, and there was no evidence of impairment of the pharmacokinetic or pharmacodynamic effects of lanadelumab. ${ }^{32}$

\section{Phase III (HELP Study, Initiated 20I6)}

A total of 125 patients were randomized and treated with subcutaneous lanadelumab $150 \mathrm{mg} / 4$ weeks, $300 \mathrm{mg} / 4$ weeks, $300 \mathrm{mg} / 2$ weeks, or placebo (lanadelumab, $\mathrm{n}=84$ vs placebo, $n=41) .{ }^{33}$ One hundred thirteen completed the 26-week study. Of the 12 patients who did not complete the study, 6 were from the lanadelumab group, 6 received placebo. One hundred and nine entered the open-label extension of the study (HELP-OLE) ${ }^{33}$

No notable safety concerns were registered during the study. Most common treatment-emergent adverse reactions (except for HAE attacks) were injection site pain (41.7\% lanadelumab vs $26.8 \%$ placebo), upper respiratory tract infections (23.8\% lanadelumab vs 26.8 placebo), headache (20.2\% lanadelumab vs $19.5 \%$ placebo), injection site bruising ( $7.1 \%$ lanadelumab vs $0 \%$ placebo), injection site erythema (9.5\% lanadelumab vs $2.4 \%$ placebo), and dizziness $(6.0 \%$ lanadelumab vs $0 \%$ placebo). There were no deaths and serious treatment-emergent adverse events.

Two patients from the placebo group withdrew from the study due to: 1) tension headache of moderate severity on day 1, and 2) a moderate HAE attack on day 12. One patient from the lanadelumab $300-\mathrm{mg} / 4$ weeks group with 
metabolic syndrome, fatty liver and multiple concomitant medications, withdrew from the study due to asymptomatic elevation of liver enzymes (AST 4.1 times the upper limit; transient) on day 139.

One patient in the $300 \mathrm{mg} / 2$ weeks group reported two hypersensitivity reactions: mouth tingling and pruritus (mild and moderate intensity, transient, no treatment was required, recovered without sequelae). The patient entered the OLE, no laboratory abnormality was detected and no antidrug antibodies were observed.

The only reported adverse events which could be speculated with higher frequency in different treatment doses were upper respiratory tract infections ( 3 subjects, $10.7 \%$ in $150 \mathrm{mg} /$ 4 weeks, $\mathrm{n}=28 ; 7$ subjects, $24.1 \%$ in $300 \mathrm{mg} / 4$ weeks, $\mathrm{n}=29 ; 10$ subjects, $37.0 \%$ in $300 \mathrm{mg} / 2$ weeks, $\mathrm{n}=27$; vs $11,26.8 \%$ in placebo group, $\mathrm{n}=41$ ), and headache ( 3 subjects, $10.7 \%$ in 150 $\mathrm{mg} / 4$ weeks; 5 subjects, $17.2 \%$ in $300 \mathrm{mg} / 4$ weeks; 9 subjects, $33.3 \%$ in $300 \mathrm{mg} / 2$ weeks; vs $8,19.5 \%$ in placebo group). Yet, the overall reported incidence of those adverse events is comparable to that of the placebo-treated patients.

Safety results from the HELP-OLE (NCT02741596, initiated 2016, estimated completion Nov 2019) are generally consistent with those from HELP study. [https:// www.int.takhzyro.com/downloads/pdf/TAKHZYRO International EU SmPC November 2018.pdf]

\section{Antidrug Antibodies}

In the HELP study 10 of 84 lanadelumab treated (11.9\%), and 2 of 41 placebo treated (4.9\%) were tested positive for antidrug antibodies (low-titer). The antidrug antibodies elevation was reported as transient in 2 of the 10 lanadelumab-treated, and 1 of 2 placebo-treated. In 3 patients from the lanadelumab group, low preexisting antibody titers were observed. This phenomenon is hypothesized to be due to the high sensitivity of the assay, and the potential for false-positive results. Neutralizing antibodies were detected close to the end of study treatment period in 2 patients from the $150 \mathrm{mg} / 4$ weeks group (reported as transient phenomenon). ${ }^{33}$ The main features of the Phase $\mathrm{Ia}, \mathrm{Ib}$ and III are summarized in Table 1.

\section{Laboratory Findings On Activated Partial Thromboplastin Time (aPTT)}

Shortened activated partial thromboplastin time (aPTT) has been previously reported in patients with $\mathrm{C} 1-\mathrm{INH}-\mathrm{HAE}$ and is assumed to be due to the higher levels of constitutive activation of Factor 12 and the dysequilibrium of the kinin- kallikrein system. ${ }^{41}$ Inhibition of kallikrein can prolong aPTT: the reagents used in the aPTT laboratory test initiate intrinsic coagulation through the activation of plasma kallikrein in the contact system. Thus, inhibition of plasma kallikrein by lanadelumab can increase aPTT in the assay. Reported was that during the HELP trial lanadelumab-treated patients had increased aPTT values with higher frequency than those receiving placebo. At baseline, mean aPTT was mildly shortened in all subgroups, relative to the normal range (20.6-39.9 s), in line with expected findings for patients with C1-INH-HAE. Activated PTT was increased from baseline to end of treatment (day 182) in patients treated with lanadelumab $300 \mathrm{mg} / 2$ weeks versus baseline. Patients in the $300 \mathrm{mg} / 4$ weeks and $150 \mathrm{mg} / 4$ weeks cohorts had increased aPTT from baseline at days 56-182 and 56-140, respectively. The majority of lanadelumab-treated patients reached the normal range after drug discontinuation. None of the increased aPTT in patients treated with lanadelumab was associated with abnormal bleeding, or thromboembolic adverse events. There were no differences in international normalised ratio (INR) and prothrombin time (PT) between treatment groups. ${ }^{33,42}$

Overall, the extent of aPTT prolongation in lanadelumab-treated patients with C1-INH-HAE is less than that observed in complete prekallikrein deficiency (Fletcher disease), supporting the evidence for incomplete suppression of plasma kallikrein activity by lanadelumab in vivo, as demonstrated by cleaved HMWK values. Based on these data, lanadelumab inhibition of plasma kallikrein activity at therapeutic doses for HAE prophylaxis theoretically does not result in clinically relevant alteration of aPTT. ${ }^{42}$

\section{Lanadelumab And Cardiovascular Risk}

Recent analysis from the HELP study showed decreased systolic and diastolic blood pressure, over time in patients receiving placebo, versus low increase in patients receiving lanadelumab. As reported, lanadelumab treatment did not increase the use of hypertension medication when compared to placebo. No important safety signals were identified in terms of mean changes from baseline in blood pressure and heart rate, and no clinically relevant ECG abnormalities were observed with lanadelumab versus placebo. ${ }^{33,43}$

\section{Lanadelumab And Pregnancy}

No information is available on the clinical use of lanadelumab during pregnancy. [https://www.int.takhzyro.com/ downloads/pdf/TAKHZYRO_International_EU_SmPC_ November 2018.pdf]. 
Table I Comparison Of The Phase la/lb And Phase III (HELP And HELP OLE) Studies

\begin{tabular}{|c|c|c|c|c|}
\hline & Phase la & Phase lb & Phase III HELP & Phase III HELP OLE \\
\hline $\begin{array}{l}\text { Sample } \\
\text { size }\end{array}$ & $N=32$ & $\begin{array}{l}\text { Total } \mathrm{N}=37 \\
\mathrm{~N}=24 \text { lanadelumab } \\
\mathrm{N}=13 \text { placebo }\end{array}$ & $\begin{array}{l}\text { Total } \mathrm{N}=125 \\
\mathrm{~N}=84 \text { lanadelumab } \\
\mathrm{N}=4 \mid \text { placebo }\end{array}$ & Ongoing \\
\hline Population & Healthy controls & $\mathrm{CI}-\mathrm{INH}-\mathrm{HAE}$ patients & $\mathrm{CI}-\mathrm{INH}-\mathrm{HAE}$ patients & $\begin{array}{l}\text { Patients who completed the double- } \\
\text { blind HELP study ("rollover } \\
\text { patients") and those who did not } \\
\text { participate in the double-blind study } \\
\text { ("non-rollover patients") }\end{array}$ \\
\hline Dose & $0.1,0.3,1$, or $3 \mathrm{mg} / \mathrm{kg}$ & $\begin{array}{l}30,100,300,400 \mathrm{mg} \text { or } \\
\text { placebo }\end{array}$ & $\begin{array}{l}\text { I50 mg every } 4 \text { weeks }(n=28) \text {, } \\
300 \text { mg every } 4 \text { weeks }(n=29) \text {, or } \\
300 \text { mg every } 2 \text { weeks }(n=27)\end{array}$ & $\begin{array}{l}\text { Rollover patients: a single } 300 \mathrm{mg} \\
\text { dose of lanadelumab on Day } 0 \text { and } \\
\text { the second dose after the patient's } \\
\text { first confirmed angioedema attack. } \\
\text { Thereafter, lanadelumab will be } \\
\text { administered every } 2 \text { weeks. Non- } \\
\text { rollover patients: } 300 \mathrm{mg} \\
\text { lanadelumab every } 2 \text { weeks } \\
\text { regardless of the first attack. }\end{array}$ \\
\hline Design & $\begin{array}{l}\text { Double-blind placebo- } \\
\text { controlled single- } \\
\text { center trial }\end{array}$ & $\begin{array}{l}\text { Randomized, multicenter, } \\
\text { double-blind, placebo- } \\
\text { controlled, multiple ascending } \\
\text { dose }\end{array}$ & $\begin{array}{l}\text { Randomized, double-blind, } \\
\text { multicenter, parallel-group, } \\
\text { placebo-controlled trial }\end{array}$ & Open-label extension \\
\hline $\begin{array}{l}\text { Main } \\
\text { results }\end{array}$ & $\begin{array}{l}\text { Prevented proteolysis } \\
\text { of } \mathrm{HMWK} \text { at the dose } \\
\text { of } 3 \mathrm{mg} / \mathrm{kg} \text {, PK } \\
\text { inhibition at the dose } \\
\text { of I and } 3 \mathrm{mg} / \mathrm{kg}\end{array}$ & $\begin{array}{l}\text { Prevented proteolysis of } \\
\text { HMWK at the dose of } 300 \\
\text { and } 400 \mathrm{mg} \text {. At the dose of } \\
100,300 \text { and } 400 \mathrm{mg} \\
\text { significantly inhibited PK. } \\
\text { Mean half-life across all the } \\
\text { groups was I3.8-15 days }\end{array}$ & $\begin{array}{l}\text { Mean difference in attack rate per } \\
\text { month in the lanadelumab groups vs } \\
\text { the placebo group of }-1.49(95 \% \mathrm{Cl} \text {, } \\
-1.90 \text { to }-1.08) \text { in the } 150-\mathrm{mg} \\
\text { every-4-week group, }-1.44(95 \% \mathrm{Cl} \text {, } \\
-1.84 \text { to }-1.04) \text { in the } 300-\mathrm{mg} \\
\text { every-4-week group, and }-1.7 \mathrm{I} \\
(95 \% \mathrm{Cl},-2.09 \text { to }-1.33) \text { in the } 300- \\
\mathrm{mg} \text { every-2-week group }\end{array}$ & Ongoing \\
\hline $\begin{array}{l}\text { Adverse } \\
\text { events }\end{array}$ & $\begin{array}{l}\text { No serious } \\
\text { No deaths Headache } \\
\text { and injection site pain } \\
\text { most commonly } \\
\text { reported }\end{array}$ & $\begin{array}{l}\text { No serious } \\
\text { No deaths } \\
\text { Headache and injection site } \\
\text { pain most commonly } \\
\text { reported. } \\
\text { Antidrug antibodies developed } \\
\text { in two patients without impact } \\
\text { on the pharmacokinetic and } \\
\text { pharmacodynamic profile }\end{array}$ & $\begin{array}{l}\text { No serious } \\
\text { No deaths } \\
\text { Headache and injection site pain } \\
\text { or erythema, upper respiratory } \\
\text { tract infections, dizziness. } \\
\text { One asymptomatic elevation of } \\
\text { liver enzymes } \\
\text { One hypersensitivity reactions. } \\
\text { Antidrug antibodies } 11.9 \% \text { in the } \\
\text { lanadelumab treated and } 4.9 \% \text { in } \\
\text { the placebo treated at low-titer }\end{array}$ & Ongoing \\
\hline
\end{tabular}

Abbreviations: HELP, Hereditary Angioedema Long-term Prophylaxis; HELP OLE, Hereditary Angioedema Long-term Prophylaxis Open-Label Extension; CI-INH-HAE, $\mathrm{Cl}$ Inhibitor Hereditary Angioedema; HMWK, High Molecular Weight Kininogen; PK, Plasma Kallikrein.

\section{Lanadelumab And Breastfeeding}

No information is available on the clinical use of lanadelumab during breastfeeding. Lanadelumab is a large protein molecule with a molecular weight of about 146000 , thus the amount in milk is likely to be very low and absorption is unlikely because it is probably destroyed in the infant's gastrointestinal tract if ever excreted with the milk. $^{44}$ 


\section{Commentary}

Inhibition of plasma kallikrein by lanadelumab seems to be effective and safe as demonstrated by the data from clinical studies, further evidence is growing since more patients become exposed to kallikrein inhibition in the clinical practice. The little data available from knowledge on Fletcher disease (congenital deficiency of prekallikrein; 81 cases reported in the literature since the disease recognition in 1965 by Dr. William Hathaway ${ }^{44}$ ) support that kallikrein deficiency seems to show no tendency of excessive bleeding ( 6 from 81 cases have been reported for such episodes), despite the prolonged aPTT. Same can be discussed about the tendency for thromboembolic events in patients with genetically deficient prekallikrein (9 of 81 patients experienced one or more such episodes). Three cases have been reported for associated Grave's disease, and one underwent thyroidectomy (no reason specified). No interpretation can be made to now as these cases might be sporadic, or with no relevant clinical association. Furthermore, bias of patient reporting must be taken into account as families with Fletcher disease have been generally neither recognized, nor reported, or maybe reported exclusively for concomitant conditions/states normally found in the general population. ${ }^{45}$ Nevertheless, depleting PK should be addressed as a delicate balance for influencing the "seamless web of host defense reactions" (the contact system), as prekallikrein-kallikrein axis plays a major regulator of Factor XII activation and BK formation with all their physiological roles (Ratnoff 1969). Further clinical experience will allow to evaluate the long-term safety for such an approach in prophylaxis of angioedema attacks in C1-INH deficient patients.

Regarding the cardiovascular system the development of hypertension and the observation of major cardiovascular events requires longer follow-up periods and higher sample size in studies designed ad hoc. The data now available do not allow to draw any definitive conclusion about the safety of the drugs on this topic.

\section{What Is The Place Of Lanadelumab In Therapy?}

Lanadelumab is a promising medication for the prophylactic treatment for the management of C1-INH-HAE. It has been recently approved for prophylactic use with the name of Takhzyro ${ }^{\circledR}$ (Takeda) in the USA, Canada and Europe for this purpose. The drug has a high efficacy in the reduction of the occurrence of the AE attacks. Based on the results of the Phase 3 trial it enters powerfully the scenario of the options for patients characterised by a severe phenotype of the disease. Thanks to its high efficacy it reduces patients' disability and impact on daily-life. It overcomes some of the limitation of existing prophylactic medications i.e. the need for frequent dosing schedules ${ }^{31,32}$ and it enables the subcutaneous route of administration, more comfortable compared to the intravenous regimen. The fully human monoclonal antibody lanadelumab specifically inhibits plasma kallikrein, ${ }^{29}$ and provides sustained inhibition. In the short-term evaluation, the safety of the medication is convincing but concern could be raised considering the effect of long-term inhibition of plasma kallikrein that is still unknown until large clinical experience will be available. Larger studies of longer duration are ongoing in the Phase III clinical program.

The rationale for the use of lanadelumab is synthesized in Figure 2, also including a new perspective for patients with HAE with normal C1-INH that are not yet tested.

Lanadelumab is expected to be a landmark in the control of the disease but it will be crucial to individualize with even more attention the right patient to direct to this type of treatment taking into account the sustainability of costs. The availability of reliable data about the number and characteristics of attacks is the key point in the definition of the severity not only of the disease but also of the patients. In general, the development of networking activity like the HAE Global Registry (ClinicalTrials.gov NCT03828279) and the sharing of standardized high-quality information will help to address the goal of the control of the disease quickly and effectively. Lanadelumab has the perspective to virtually set to zero the disease-related mortality, but nowadays the mortality due to $\mathrm{AE}$ in the incoming countries is still unacceptable, data available from Bulgaria ${ }^{47,46}$ documents that from 1972 to 2012 the mean age at death was 36.6 years mainly due to the lack of treatment. Despite lanadelumab the first objective and priority for the control of the disease is to have the medication available.

\section{Future Directions}

While the role of lanadelumab in the field of LTP treatment is very clear for the form of HAE C1-INH deficiency the future is instead very challenging for primary angioedema with normal $\mathrm{C} 1 \mathrm{INH}$. The lack of knowledge about details on the pathogenesis of these diseases let patients without a defined therapy. Some case series describe experiences with drugs successfully used for $\mathrm{C} 1 \mathrm{INH}$ deficiency forms, but 
there are no clinical trials proving their efficacy. Due to the potential role of kallikrein in primary angioedema with normal $\mathrm{C} 1 \mathrm{INH}$, we hypothesize a possible place for lanadelumab in the prophylaxis (Figure 2).

Considering the novelty of the drug and the lack of data in a real-life context this review does not address the topic of the cost-effectiveness of the treatment that should be carefully evaluated in the next future.

\section{Disclosure}

Dr Maria Bova reports personal fees and travel grants from Shire - ora Takeda, personal fees and travel grants from CSL behring, outside the submitted work; Dr Anna Valerieva reports grants from Bulgarian Ministry of Science under the National Program for Research, personal fees from Shire/Takeda, personal fees from Pharming Group N.V., personal fees from CSL Behring, outside the submitted work; Dr Riccardo Senter reports travel grants received by Shire-Takeda, CSL Behring, Biocryst and Novartis. The authors report no other conflicts of interests in this work.

\section{References}

1. Cicardi M, Aberer W, Banerji A, et al. Classification, diagnosis and approach to treatment for angioedema: consensus report from the Hereditary Angioedema International Working Group. Allergy. 2014;69:602-616. doi:10.1111/all.12380

2. Banerji A, Busse P, Christiansen SC, et al. Current state of hereditary angioedema management: a patient survey. Allergy Asthma Proc. 2015;36(3):213-217. doi:10.2500/aap.2015.36.3824

3. Aygören-Pürsün E, Bygum A, Beusterien K, et al. Socioeconomic burden of hereditary angioedema: results from the hereditary angioedema burden of illness study in Europe. Orphanet J Rare Dis. 2014;4 (9):99. doi:10.1186/1750-1172-9-99

4. Bova M, De Feo G, Parente R, et al. Hereditary and acquired angioedema: heterogeneity of pathogenesis and clinical phenotypes. Int Arch Allergy Immunol. 2018;175(3):126-135. doi:10.1159/000486312

5. Valerieva A, Perego F, Porebski G, et al. Hereditary angioedema: treatment options and availability. Balance between patients' needs and stakeholders' plans. Alergia Astma Immunologia. 2018;23(4).

6. Blankart CR, Stargardt T, Schreyögg J. Availability of and access to orphan drugs: an international comparison of pharmaceutical treatments for pulmonary arterial hypertension, fabry disease, hereditary angioedema and chronic myeloid leukaemia. Pharmacoeconomics. 2011;29(1):63-82. doi:10.2165/11539190-000000000-00000

7. Longhurst H, Bygum A. The humanistic, societal, and pharmacoeconomic burden of angioedema. Clin Rev Allergy Immunol. 2016;51 (2):230-239. doi:10.1007/s12016-016-8575-2

8. Federici C, Perego F, Borsoi L, et al. Costs and effects of on-demand treatment of hereditary angioedema in Italy: a prospective cohort study of 167 patients. BMJ Open. 2018;8:e022291. doi:10.1136/bmjopen2018-022291

9. Aygören-Pürsün E, Magerl M, Maetzel A, et al. Epidemiology of Bradykinin-mediated angioedema: a systematic investigation of epidemiological studies. Orphanet J Rare Dis. 2018;13(1):73. doi:10.1186/ s13023-018-0822-6
10. Dewald G, Bork K. Missense mutations in the coagulation factor XII (Hageman factor) gene in hereditary angioedema with normal C1 inhibitor. Biochem Biophys Res Commun. 2006;343(4):1286-1289. doi:10.1016/j.bbrc.2006.03.092

11. Bafunno V, Firinu D, D'Apolito M, et al. Hereditary angioedema with a mutation in the plasminogen gene. J Allergy Clin Immunol. 2017;S0091-6749(17):30921-1.

12. Bork K, Wulff K, Steinmüller-Magin L, et al. Hereditary angioedema cosegregating with a novel kininogen 1 gene mutation changing the N-terminal cleavage site of bradykinin. Allergy. 2018;73(2):442-450. doi:10.1111/all.13270

13. Bork K, Wulff K, Rossmann H, et al. Hereditary angioedema cosegregating with a novel kininogen 1 gene mutation changing the N-terminal cleavage site of bradykinin. Allergy. 2019. doi:10.1111/ all.13869

14. Caccia S, Suffritti C, Cicardi M. Pathophysiology of hereditary angioedema. Pediatr Allergy Immunol Pulmonol. 2014;27(4):159163. doi:10.1089/ped.2014.0425

15. Cicardi M, Banerji A, Bracho F, et al. Icatibant, a new bradykininreceptor antagonist, in hereditary angioedema. $N$ Engl J Med. 2010;363(6):532-541. doi:10.1056/NEJMoa0906393

16. Bork K, Wulff K, Hardt J, et al. Hereditary angioedema caused by missense mutations in the factor XII gene: clinical features, trigger factors, and therapy. J Allergy Clin Immunol. 2009;124:129-134. doi:10.1016/j.jaci.2009.03.038

17. Bjorkqvist J, de Maat S, Lewandrowski U, et al. Defective glycosylation of coagulation factor XII underlies hereditary angioedema type III. J Clin Invest. 2015;125:3132-3146. doi:10.1172/JCI77139

18. Bafunno V, Firinu D, D'Apolito M, et al. Mutation of the angiopoietin-1 gene (ANGPT1) associates with a new type of hereditary angioedema. J Allergy Clin Immunol. 2018;141:1009-1017. doi:10. 1016/j.jaci.2017.05.020

19. Bork K, Wulff K, Steinmüller-Magin L, et al. Hereditary angioedema with a mutation in the plasminogen gene. Allergy. 2018;73:442-450. doi:10.1111/all.13270

20. Li HH, Riedl M, Kashkin J. Update on the use of C1-esterase inhibitor replacement therapy in the acute and prophylactic treatment of hereditary angioedema. Clin Rev Allergy Immunol. 2019;56:207218. doi:10.1007/s12016-018-8684-1

21. Cicardi M, Suffritti C, Perego F, Caccia S. Novelties in the diagnosis and treatment of angioedema. J Investig Allergol Clin Immunol. 2016;26(4):212-221. doi:10.18176/jiaci.0087

22. Riedl MA. Critical appraisal of androgen use in hereditary angioedema: a systematic review. Ann Allergy Asthma Immunol. 2015;114 (4):281-288. doi:10.1016/j.anai.2015.01.003

23. Riedl MA, Grivcheva-Panovska V, Moldovan D, et al. Recombinant human $\mathrm{C} 1$ esterase inhibitor for prophylaxis of hereditary angiooedema: a phase 2, multicentre, randomised, double-blind, placebocontrolled crossover trial. Lancet. 2017;390(10102):1595-1602. doi:10.1016/S0140-6736(17)31963-3

24. Aygören-Pürsün E, Bygum A, Grivcheva-Panovska V, et al. Oral plasma kallikrein inhibitor for prophylaxis in hereditary angioedema. N Engl J Med. 2018;379(4):352-362. doi:10.1056/NEJMoa1716995

25. Perego F, Wu MA, Valerieva A, et al. Current and emerging biologics for the treatment of hereditary angioedema. Expert Opin Biol Ther. 2019;19(6):517-526. doi:10.1080/14712598.2019.1595581

26. Liu J, Qin J, Borodovsky A, et al. An investigational RNAi therapeutic targeting factor XII (ALN-F12) for the treatment of hereditary angioedema. RNA. 2019;25(2):255-263. doi:10.1261/rna.068916.118

27. Qiu T, Chiuchiolo MJ, Whaley AS, et al. Gene therapy for C1 esterase inhibitor deficiency in a Murine model of hereditary angioedema. Allergy. 2019;74(6):1081-1089. doi:10.1111/all.13582

28. Bhattacharjee G, Revenko AS, Crosby JR, et al. Inhibition of vascular permeability by antisense-mediated inhibition of plasma kallikrein and coagulation factor 12. Nucleic Acid Ther. 2013;23(3):175-187. doi:10.1089/nat.2013.0417 
29. Kenniston JA, Faucette RR, Martik D, et al. Inhibition of plasma kallikrein by a highly specific active site blocking antibody. J Biol Chem. 2014;289(34):23596-23608. doi:10.1074/jbc.M114.569061

30. Hoet RM, Cohen EH, Kent RB, et al. Generation of high-affinity human antibodies by combining donor-derived and synthetic complementarity-determining-region diversity. Nat Biotechnol. 2005; 23:344-348.

31. Chyung Y, Vince B, Iarrobino R, et al. A phase 1 study investigating DX-2930 in healthy subjects. Ann Allergy Asthma Immunol. 2014;113(4):460-466. doi:10.1016/j.anai.2014.05.028

32. Banerji A, Busse P, Shennak M, et al. Inhibiting Plasma Kallikrein for hereditary angioedema prophylaxis. $N$ Engl J Med. 2017;376 (8):717-728. doi:10.1056/NEJMoa1605767

33. Banerji A, Riedl MA, Bernstein JA, et al. Effect of lanadelumab compared with placebo on prevention of hereditary angioedema attacks: a randomized clinical trial. JAMA. 2018;320(20):21082121. doi:10.1001/jama.2018.16773

34. Riedl MA, Bernstein JA, Craig T, et al. An open-label study to evaluate the long-term safety and efficacy of lanadelumab for prevention of attacks in hereditary angioedema: design of the HELP study extension. Clin Transl Allergy. 2017;7:36. doi:10.1186/s13601017-0172-9

35. Wang Y, Marier J, Kassir N, Gosselin NH, Martin P. Exposureresponse analyses of lanadelumab in patients with hereditary angioedema. J Allergy Clin Immunol. 2019;143(2):AB40. doi:10.1016/j.jaci. 2018.12.122

36. Banerji A, Manning ME, Martinez-Saguer I, et al. Subgroup analyses from the Phase 3 HELP study of lanadelumab for the prevention of hereditary angioedema attacks. J Allergy Clin Immunol. 2019;143(2): AB42. doi:10.1016/j.jaci.2018.12.126

37. Inhaber N, Zuraw BL, Marier J, et al. Relationship between body weight, PK/ PD, and attack responses following subcutaneous administration of lanadelumab in patients with hereditary angioedema. $J$ Allergy Clin Immunol. 2019;143(2):AB38. doi:10.1016/j.jaci.2018. 12.117
38. Riedl MA, Anderson JT, Cicardi M, et al. Efficacy of lanadelumab in hereditary angioedema patients switching from $\mathrm{C} 1$ inhibitor longterm prophylaxis: interim results from the HELP open-label extension study. J Allergy Clin Immunol. 2018;143(2):AB37. doi:10.1016/ j.jaci.2018.11.043

39. Jacobs JS, Bernstein J, Davis-Lorton M, et al. Increased response to higher dose lanadelumab in hereditary angioedema patients: exploratory findings from the HELP and HELP OLE studies. J Allergy Clin Immunol. 2019;143(2):AB38. doi:10.1016/j.jaci.2018.12.116

40. Busse PJ, Tachdjian R, Martinez-Saguer I, et al. Efficacy and safety of lanadelumab for prophylactic treatment in adolescents with hereditary angioedema (HAE). J Allergy Clin Immunol. 143(2):AB43. doi:10.1016/j.jaci.2018.12.131

41. Konrad Bork Günther Witzke. Shortened activated partial thromboplastin time may help in diagnosing hereditary and acquired angioedema. Int Arch Allergy Immunol. 2016;170:101-107. doi:10.1159/ 000447695

42. Schmaier AH, Bauer KA, Cicardi M, et al. Effect of lanadelumab on coagulation parameters in patients with hereditary angioedema: findings from the phase 3 HELP study. AAAI 2019 San Francisco Poster.

43. Sexton DJ, Brown N, Lumry W, et al. Lanadelumab and cardiovascular risk: findings from the phase 3 HELP study. AAAI 2019 San Francisco Poster.

44. Available from: https://www.int.takhzyro.com/downloads/pdf/ TAKHZYRO International_EU_SmPC November_2018.pdf.

45. Hathaway WE, Belhasen LP, Hathaway HS. Evidence for a new plasma thromboplastin factor. 1. Case report, coagulation studies, and physicochemical properties. Blood. 1965;26:521-532.

46. Saito H. Studies on Fletcher trait and Fitzgerald trait. A rare chance to disclose body's defense reactions against injury. Thromb Haemost. 2010;104(05):867-874. doi:10.1160/TH10-01-0058

47. Valerieva A, Petkova E, Suffritti C Rare forms of angioedema in bulgaria: experience since 1972. Past And Present. EAACI 2019, Lisbon, Poster.

\section{Publish your work in this journal}

Drug Design, Development and Therapy is an international, peerreviewed open-access journal that spans the spectrum of drug design and development through to clinical applications. Clinical outcomes, patient safety, and programs for the development and effective, safe, and sustained use of medicines are a feature of the journal, which has also been accepted for indexing on PubMed Central. The manuscript management system is completely online and includes a very quick and fair peer-review system, which is all easy to use. Visit http://www. dovepress.com/testimonials.php to read real quotes from published authors. 